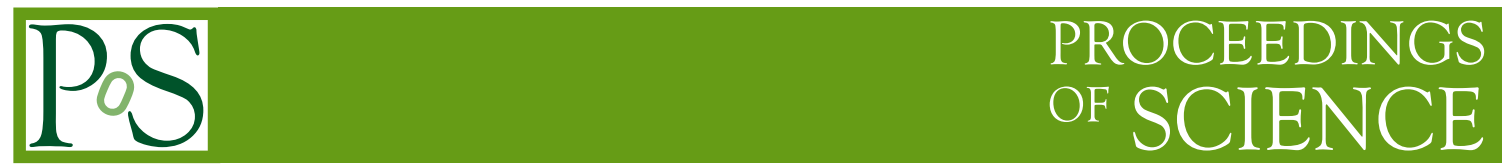

\title{
A new vision of the inert doublet model of dark matter
}

\section{Laura Lopez-Honorez*Universidad Autónoma de Madrid \& Université Libre de Bruxelles}

The results presented in these proceedings were originaly obtained in collaboration with

Carlos E. Yaguna in Ref. [1]

E-mail: llopezho@ulb.ac.be

The Inert Doublet Model (IDM) is a simple and yet very rich extension of the Standard Model which provides interresting scalar dark matter candidates. In these proceedings, we show that annihilation into 3 body final states $W W^{*} \rightarrow \bar{f} f^{\prime}$, overlooked in all the previous studies devoted to this model, can significantly affect the viable parameter space of the IDM as well as the prospects for direct and indirect detection searches.

Identification of Dark Matter 2010-IDM2010

July 26-30, 2010

Montpellier France

\footnotetext{
* Speaker.
} 

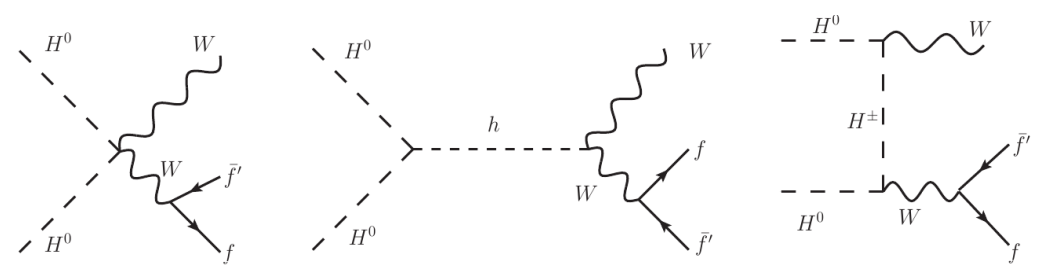

Figure 1: The Feynman diagrams that contribute, in the unitary gauge, to the $H^{0} H^{0}$ annihilation into the three-body final state $W W^{*} \rightarrow W f \bar{f}^{\prime}$ within the inert doublet model.

\section{Introduction}

Even though dark matter accounts for about $23 \%$ of the energy density of the Universe [2], we do not yet know its true substance. Among the zoo of dark matter candidates now available in the literature, the inert dark matter particle has earned a special place as a representative candidate of weakly interacting scalar dark matter.

In the inert doublet model, a higgs doublet $H_{2}$, odd under a new $Z_{2}$ symmetry, is added to the standard model particle content. The scalar potential of this model is given by

$$
V=\mu_{1}^{2}\left|H_{1}\right|^{2}+\mu_{2}^{2}\left|H_{2}^{2}\right|+\lambda_{1}\left|H_{1}\right|^{4}+\lambda_{2}\left|H_{2}\right|^{4}+\lambda_{3}\left|H_{1}\right|^{2}\left|H_{2}\right|^{2}+\lambda_{4}\left|H_{1}^{\dagger} H_{2}\right|^{2}+\frac{\lambda_{5}}{2}\left[\left(H_{1}^{\dagger} H_{2}\right)^{2}+\text { h.c. }\right],
$$

where $H_{1}$ is the Brout- Englert- Higgs doublet (refered as higgs in the following), and $\lambda_{i}$ and $\mu_{i}$ are real parameters. Four new physical states are obtained in this model: two charged states, $H^{ \pm}$, and two neutral ones, $H^{0}$ and $A^{0}$. We choose $H^{0}$ to be the lightest inert particle, $m_{H^{0}}^{2}<m_{A^{0}}^{2}, m_{H^{ \pm}}^{2}$ and the dark matter candidate. In our study, we will use the following free paremeters: the combination $\lambda_{L}=\left(\lambda_{3}+\lambda_{4}+\lambda_{5}\right) / 2$ corresponding to the scalar coupling of a pair of $H_{0}$ to the higgs particle $h$; $m_{H^{0}}$, the $H_{0}$ mass; $\Delta m_{A^{0}}=m_{A^{0}}-m_{H^{0}}$ and $\Delta m_{H^{ \pm}}=m_{H^{ \pm}}-m_{H^{0}}$, two mass splittings between the inert scalars, and the higgs mass, $m_{h}$. Notice that the $\lambda_{2}$ parameter has a small impact on the dark matter analysis. We take into account all the known theoretical and experimental constraints on this model -see [3] and [4]. This model has been extensively studied in a number of recent works (see [1] and references therein). It was shown that the dark matter relic density constraint can be satisfied for restricted values of $m_{H^{0}}$. Four viable regions can be distinguished: a small mass regime with $m_{H^{0}} \sim 8 \mathrm{GeV}[5,6]$, a large mass regime with $m_{H_{0}}>500 \mathrm{GeV}[4,7,8]$ and two intermediate mass regime: $m_{H_{0}} \lesssim M_{W}[3,4]$ and $m_{H_{0}} \gtrsim M_{W}$ (as recently pointed out in [9]).

On general grounds annihilation of dark matter particles can receive large contributions from three-body final states consisting of a real and a virtual massive particle [10-12]. In [1], we pointed out that the annihilation into the three-body final state $W W^{*}\left(\rightarrow W f \bar{f}^{\prime}\right)$, are important in the intermediate mass region below the $W$ threshold. In these proceedings, we summarize the impact of the inclusion of the three-body final state $W W^{*}$ on the IDM.

\section{Impact of the $W W^{*}$ annihilation processes for fixed parameters}

In order clarify how important the annihilation of dark matter into $W W^{*}\left(H^{0} H^{0} \rightarrow W W^{*} \rightarrow\right.$ $W f \bar{f}^{\prime}$ ) can be in the inert doublet model, we first focus on the three-body annihilation cross-section 
$\sigma\left(H^{0} H^{0} \rightarrow W W^{*}\right)$ fixing the free parameters of the model without imposing the WMAP relic abundance constraint [2]. In figure 1, we represent the three diagrams contributing to the annihilation of dark matter into $W W^{*}$. For the range of parameter that we consider here $\left(m_{H_{0}}<m_{A_{0}}, m_{H_{ \pm}}\right.$and $m_{H_{0}} \lesssim M_{W}$ ), their amplitudes depends weakly on $m_{A^{0}}$ (only through the higgs width) and on $m_{H^{+}}$ (the $H^{+}$mediated diagram is suppressed by the $\mathrm{t}$-(u-)channel propagator). $\sigma\left(H^{0} H^{0} \rightarrow W W^{*}\right)$ is however much more sensitive to $m_{H^{0}}, \lambda_{L}$ (sign and magnitude), and $m_{h}$.

In figure 2, the left panels compare the two- and three-body annihilation rates at low velocity, denoted by $\sigma \mathrm{v}$, for three different higgs masses $m_{h}=120$ (top), 150 (middle) and 200 (bottom) GeV. The two-body annihilation rate that has been thought to drive indirect detection processes (well) below the $W$ threshold is $H^{0} H^{0} \rightarrow h \rightarrow f \bar{f}$. It is a higgs mediated process, which amplitude depends on $\lambda_{L}$ and on the Yukawa coupling of the outgoing fermions $f$ to the higgs. We see in figure 2 that the three-body process can actually compete with the two-body ones. This is related to the Yukawa suppression present in $\sigma \mathrm{v}_{2 \text {-body }}$ and to the large multiplicity of final states associated with $W W^{*}\left(\rightarrow \sum_{f} W f \bar{f}^{\prime}\right)$ processes. $\sigma \mathrm{v}_{3}$-body generically increases as $m_{H^{0}}$ gets closer to $M_{W}$ and its dependence in the scalar parameters $\lambda_{L}, m_{h}$ is stronger around the higgs resonance, $m_{H^{0}} \sim m_{h} / 2$. More specifically, the presence of a trough in $\sigma v_{3-\text { body }}$ next to $m_{H^{0}}=m_{h} / 2$ is due to the interference between the purely gauge diagram and the higgs mediated diagram (left and central diagrams in fig. 1). Because of such interference, the three-body cross section for $\lambda_{L}>0$ (dash-dotted line) is larger than that for $\lambda_{L}<0$ (dashed line) above the higgs resonance but smaller than it below the resonance. In any case, the crucial point for us is that the three-body cross section is not negligible at all, especially next to the $W$ threshold.

We can now compare the relic density obtained for two-body final states only (denoted as $\Omega\left(2\right.$ - body)) with that predicted including also the final state $W W^{*}$ (denoted as $\Omega(3$ - body) and referred to as the 3-body relic density). Lets us mentions that for our calculations, we have used a modified version of micrOMEGAs, see [13] and references therein, in which we incorporated the annihilation into the three-body final state $W W^{*}$. To illustrate the effect of the three-body final state on the relic abundance, we show in the right panels of figure 2 the ratio $\Omega(3-$ body $) / \Omega(2$ - body $)$ as a function of $m_{H_{0}}$ for three values of the higgs masses $m_{h}=120$ (top), 150 (middle) and 200 (bottom) GeV. In each plots, we have represented $\Omega$ (3-body) $/ \Omega(2$ - body) for two values of $\Delta m_{A_{0}}=10,50 \mathrm{GeV}$ in order to illustrate the effect of coannihilations.

A ratio equal to 1 means that the three-body process gives a negligible correction to the calculation of the relic density. Clearly, that is not the case. The ratio tends to 1 for $m_{H^{0}}$ close to $M_{W}$, where the annihilation into $W^{+} W^{-}$is efficient, and for $m_{H^{0}} \ll M_{W}$, where the three-body annihilation is suppressed, but in the intermediate region the three-body final state plays a major role, giving rise to a correct relic density significantly smaller than the two-body one. An effect that is present for every higgs mass and can lead to an overestimation of the predicted relic density by more than one order of magnitude. Notice that using smaller $\Delta m_{A^{0}}$, the coannihilation through the process $H^{0} A^{0} \rightarrow Z^{*} \rightarrow f \bar{f}^{\prime}$ increases the effective annihilation rate that drives the relic abundance. They also reduce the impact of three-body process on the relic density. Indeed, in the right panels of figure 2, we see that the ratio $\Omega(3-$ body $) / \Omega(2-$ body $)$ is usually less suppressed for $\Delta m_{A^{0}}=10$ $\mathrm{GeV}$ (when coannihilations are important) than for $\Delta m_{A^{0}}=50 \mathrm{GeV}$. Although coannihilation effects slightly reduce its relevance, the effect of the three-body final state remains important over a significant portion of the viable parameter space of the inert doublet model. 

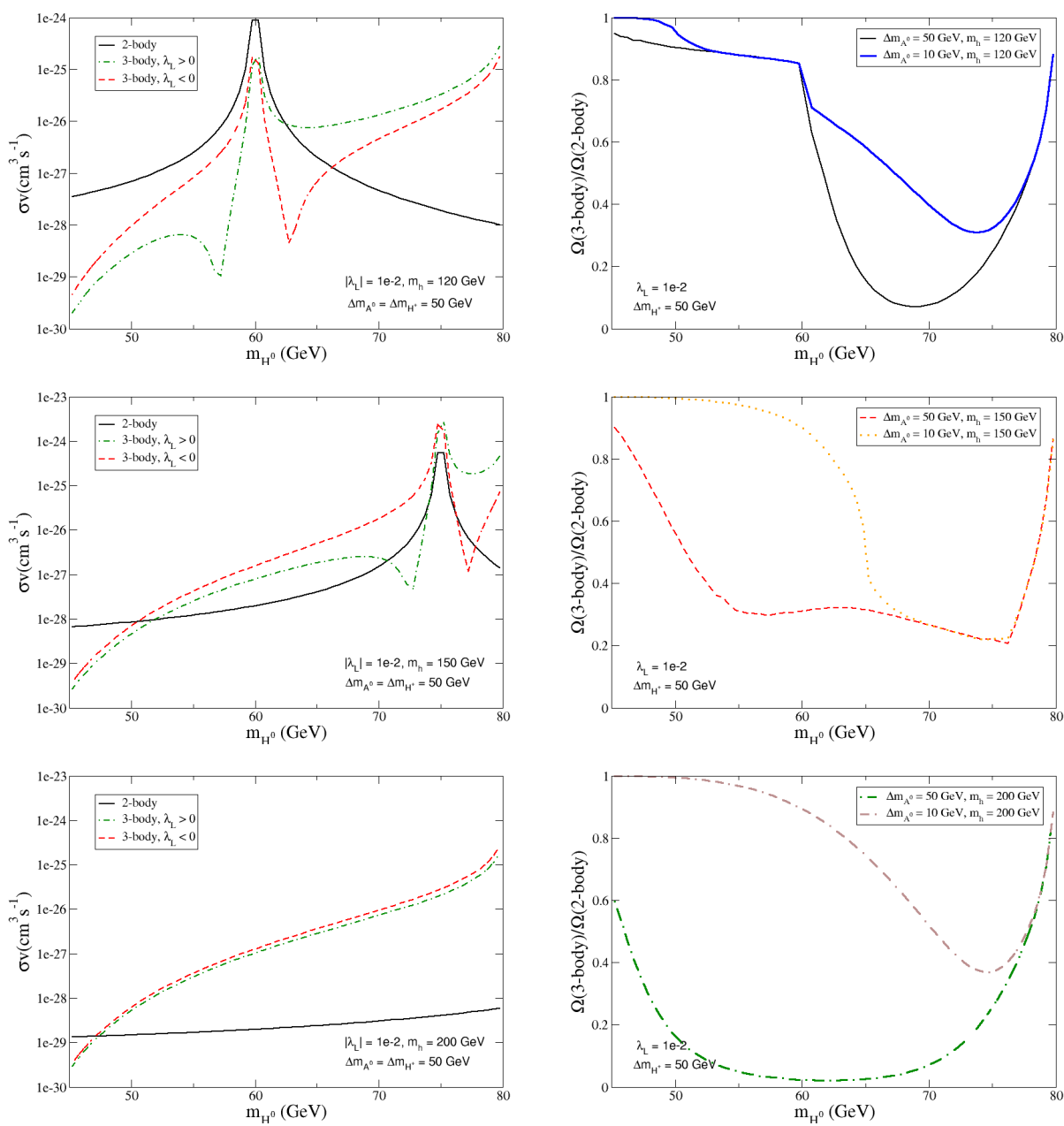

Figure 2: Annihilation rates and relic density ratios as a function of $m_{H_{0}}$ for $\left|\lambda_{L}\right|=10^{-2}$ and from top to bottom for $m_{h}=120,150$ and $200 \mathrm{GeV}$. Left Panels: Comparison between the three-body and the two-body annihilation rate, $\sigma \mathrm{v}$, as a function of the dark matter mass for the two possible signs of $\lambda_{L}$. The other parameters were taken as $\Delta m_{A^{0}}=\Delta m_{H^{ \pm}}=50 \mathrm{GeV}$. Right panels: Ratio between the relic density including the three-body final state and the relic density for two-body final states only. $\Delta m_{A^{0}}$ affects $\Omega h^{2}$ through coannihilations effects, which are important for small mass splittings $\left(\Delta m_{A^{0}}=10 \mathrm{GeV}\right)$ but not for large ones $\left(\Delta m_{A^{0}}=50 \mathrm{GeV}\right)$.

\section{The Impact of $W W^{*}$ on the viable parameter space}

We can now study the impact of the three-body process on the viable parameter space i.e. the parameter space determined by requiring that the predicted relic abundance be compatible with the observed density of dark matter [2]. For definiteness, we focus on the following three interesting cases: $m_{h}=120 \mathrm{GeV}$ with $\lambda_{L}>0, m_{h}=150 \mathrm{GeV}$ with $\lambda_{L}<0$, and $m_{h}=200 \mathrm{GeV}$ with $\lambda_{L}<0$. The left panels of figure 3 shows the viable parameter space of the intermediate mass range of the inert dark matter model in the plane $\left(\lambda_{L}, m_{H^{0}}\right)$ for $\Delta m_{H^{ \pm}}=50 \mathrm{GeV}$, and two different values of $\Delta m_{A_{0}}$, $10 \mathrm{GeV}$ (more coannihilations) and $50 \mathrm{GeV}$. The thin lines in these figures correspond to the viable 

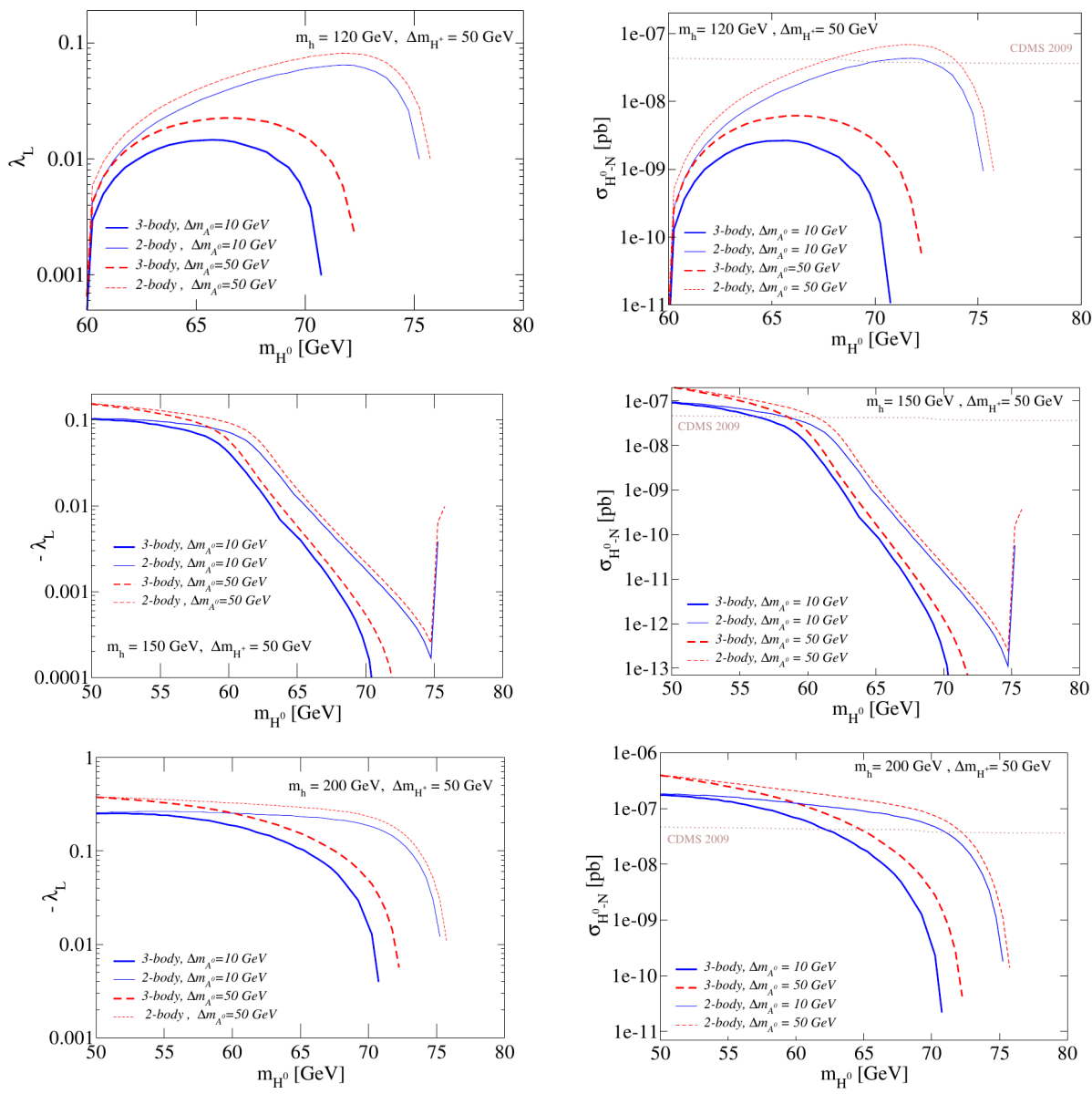

Figure 3: The viable parameter space for $m_{h}=150 \mathrm{GeV}$ (left panel) and $m_{h}=200 \mathrm{GeV}$ (right panel). Along the lines $\Omega h^{2}=0.11$. The thick lines are the result including the final state $W W^{*}$, the thin lines correspond to 2-body final states only.

regions if only two-body final states are considered. The thick lines, on the contrary, correspond to the genuine viable regions, those obtained by taking into account two- and three-body final states in the calculation of the relic density. We see that, as a consequence of the three-body final state contribution to the annihilation rate of inert higgs dark matter, the required value of $\lambda_{L}$ is smaller at any given mass, and the maximum allowed value of $m_{H^{0}}$ gets reduced by several GeVs (notice that here $\mu_{2}^{2}>0$ only has been considered). The modification of the viable parameter space, induced by the annihilation into the three-body final state $W W^{*}$, appears to be a generic feature of the inert doublet model. A feature that is present over a wide range of $m_{H^{0}}$ quite independently of the other parameters of the model. As a consequence, the prospects for direct, indirect detection but also higgs searches have to be reexamined.

In the inert higgs model, the $H^{0} N$ scattering cross section, $\sigma_{H^{0} N}$, relevant for direct detection is higgs-mediated and is proportional to $\lambda_{L}^{2}$. Given the new allowed values of $\lambda_{L}$ that were derived above, $\sigma_{H^{0} N}$ appears to be significantly reduced with respect to the two-body result used, until now, in the literature. This is illustrated in the right panels of figure 3 where the prediction for $\sigma_{H^{0} N}$ are 

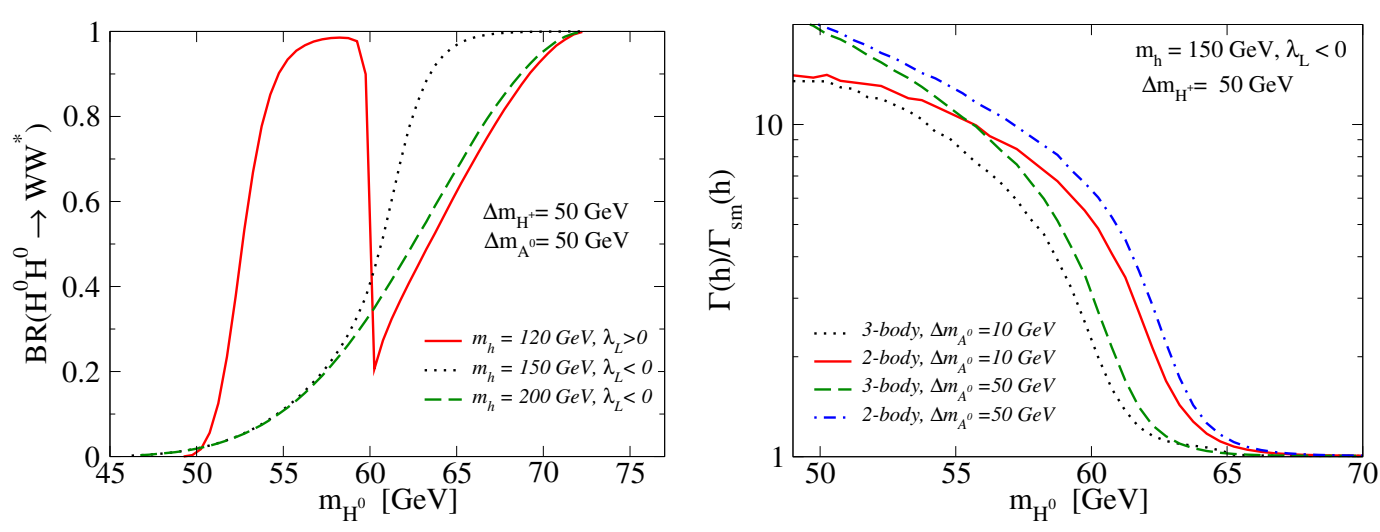

Figure 4: Left Panel: Annihilation branching ratio into the three-body final state $W W^{*}$ along the viable regions of the inert doublet model. Right Panel: Ratio between the higgs branching ratios in the inert doublet model and in the standard model along the viable regions for $m_{h}=150 \mathrm{GeV}$. $\lambda_{L}$ was taken to be negative and $\Delta m_{H^{ \pm}}=50 \mathrm{GeV}$.

shown along the viable lines of the inert doublet model for $m_{h}=120$ (top), 150 (middle) and 200 (bottom) GeV. For comparison, in this figure we also show, as a dotted line, the current limit from CDMS [14]. Notice from the figure that the correct direct detection cross section can be more than two orders of magnitude smaller than the one obtained for two-body final states leading to less stringent constraints on the IDM from the present bounds set by direct detection searches.

The indirect detection signals of inert higgs dark matter are also altered by the existence of the three-body final state $W W^{*}$. On the one hand, these signals should be now computed along new regions, due to the modified viable parameter space. On the other hand, in these new regions the annihilation cross section and branching ratios typically receive large corrections from the threebody final state $W W^{*}$. As a result, the spectrum of photons, neutrinos, positrons and antiprotons expected from inert higgs annihilation will be different, changing its indirect detection prospects. In the left panel of figure 4 , we show that the three-body final state $W W^{*}$ becomes dominant over a sizeable region of the viable parameter space.

In the inert doublet model, the higgs boson can decay also into $H^{0} H^{0}$ and $A^{0} A^{0}$, increasing the higgs decay width and modifying its branching ratios. The contribution to the higgs decay with from the decay into the inert scalars is proportional to $\lambda_{L}^{2}$, so that it will be affected by the three-body final state $W W^{*}$ via the new viable parameter space. In the right panel of figure 4, we illustrate for $m_{h}=150 \mathrm{GeV}$ how the higgs decay width can be modified when including the threebody final state in the determination of the relic abundance. This should be taken into account for higgs searches at colliders.

\section{Conclusions}

We studied the impact, on the phenomenology of the inert doublet model, of dark matter annihilation into the three-body final state $W W^{*}$. The annihilation cross section into $W W^{*}, \sigma\left(H^{0} H^{0} \rightarrow\right.$ $\left.W W^{*}\right)$, was shown to dominate the total dark matter annihilation cross section over a relevant portion of the parameter space. In consequence, the predicted relic density differs considerably from 
that found in earlier works. The genuine viable parameter space of the inert doublet model is clearly affected by three-body process. Including the latter in the derivation of the relic density, the viable $H_{0}$-coupling to the higgs $\left(\lambda_{L}\right)$ can be reduced by one order of magnitude. This implies that the scattering cross section $\left(\propto \lambda_{L}^{2}\right)$ relevant for direct detection searches can become two orders of magnitude smaller. In these proceedings, we also briefly considered some implications of these new annihilation processes on the decay width of the higgs boson and on the indirect detection of inert higgs dark matter.

Acknowledgments: I would like to thank the organizers of IDM 2010 for giving me the opportunity to present this work at the conference. I was partially supported by CICYT through the project FPA2009-09017, by CAM through the project HEPHACOS, P-ESP-00346, by the PAU (Physics of the accelerating universe) Consolider Ingenio 2010, by the F.N.R.S. and the I.I.S.N..

\section{References}

[1] Laura Lopez Honorez and Carlos E. Yaguna. The inert doublet model of dark matter revisited. JHEP, 09:046, 2010.

[2] E. Komatsu et al. Five-Year Wilkinson Microwave Anisotropy Probe (WMAP 1 ) Observations:Cosmological Interpretation. Astrophys. J. Suppl., 180:330-376, 2009.

[3] Riccardo Barbieri, Lawrence J. Hall, and Vyacheslav S. Rychkov. Improved naturalness with a heavy Higgs: An alternative road to LHC physics. Phys. Rev., D74:015007, 2006.

[4] Laura Lopez Honorez, Emmanuel Nezri, Josep F. Oliver, and Michel H. G. Tytgat. The inert doublet model: An archetype for dark matter. JCAP, 0702:028, 2007.

[5] Thomas Hambye and Michel H. G. Tytgat. Electroweak Symmetry Breaking induced by Dark Matter. Phys. Lett., B659:651-655, 2008.

[6] Sarah Andreas, Thomas Hambye, and Michel H. G. Tytgat. WIMP dark matter, Higgs exchange and DAMA. JCAP, 0810:034, 2008.

[7] Marco Cirelli, Nicolao Fornengo, and Alessandro Strumia. Minimal dark matter. Nucl. Phys., B753:178-194, 2006.

[8] T. Hambye, F. S. Ling, L. Lopez Honorez, and J. Rocher. Scalar Multiplet Dark Matter. JHEP, 07:090, 2009.

[9] Laura Lopez-Honorez and Carlos E. Yaguna. A new viable region of the inert doublet model. 2010.

[10] Carlos E. Yaguna. Large contributions to dark matter annihilation from three-body final states. Phys. Rev., D81:075024, 2010.

[11] Xue-lei Chen and Marc Kamionkowski. Three-body annihilation of neutralinos below two-body thresholds. JHEP, 07:001, 1998.

[12] Yutaka Hosotani, Pyungwon Ko, and Minoru Tanaka. Stable Higgs Bosons as Cold Dark Matter. Phys. Lett., B680:179-183, 2009.

[13] G. Belanger, F. Boudjema, A. Pukhov, and A. Semenov. Dark matter direct detection rate in a generic model with micrOMEGAs2.1. Comput. Phys. Commun., 180:747-767, 2009.

[14] Z. Ahmed et al. Results from the Final Exposure of the CDMS II Experiment. 2009. 